\title{
PRINCIPAL COMPONENT ANALYSIS OF MULTIWAVELENGTH PROPERTIES OF SEYFERT GALAXIES
}

\author{
DEBORAH DULTZIN-HACYAN and CARLOS RUANO \\ Instituto de Astronomía UNAM, Apdo 70-264 Mexico DF 04510, Mexico
}

\begin{abstract}
A multidimensional statistical analysis of observed properties of Seyfert galaxies has been carried out using Principal Component Analysis (PCA) applied to X-ray, optical, near and far IR and radio data for all the Seyfert galaxies types 1 and 2 for the catalog by Lipovtsky et al. (1987).
\end{abstract}

Key words: Seyfert galaxies, multiwavelength emission, multidimensional statistics

\section{Analysis and Results}

The catalog by Lipovetsky et al. (1987) provides the largest list of Seyferts (nearly 1000) with multiwavelength data. The effects of incompleteness of the sample as well as other details of the statistics and interpretation will be discussed elsewhere (Dultzin-Hacyan \& Ruano, in preparation). PCA is a statistical method which permits to determine the minimum number of independent or uncorrelated variables underlying a larger number of observed variables, see e.g. Kendall (1957). Dimensional analysis is a powerful tool in studies that require classification and is very important for understanding correlations among different variables.

Our main result is that the emission of Seyfert 1 galaxies is well represented by only one eigenvector; i.e., the observed quantities generate a one-dimensional space of variables. On the other hand, in the case of Seyfert 2 galaxies, the space of variables generated by the PCA is three-dimensional. Several details of the analysis lead us to the following interpretation of this result: In the case of Seyferts 1 , the main process at the origin of radiation is the release of energy of gravitational origin by accretion unto a supermassive black hole. All the considered luminosities (even if they are the result of re-processing of this energy) are thus coupled to the intensity of this process. In the case of Seyferts 2, appart from accretion energy, there are two other important processes which we may identify with stellar and interstellar radiation from the underlying galaxy. A detailed analysis reveals that the variance in luminosity related to radiation of stellar origin in no case exceeds $\sim 13 \%$ for Seyferts 1 . In contrast, for Seyferts 2 the radiation of stellar origin can account for $\sim 46 \%$ of the variance in certain luminosities.

\section{References}

Lipovetsky, V.A., Neisvestny, S.I. \& Neizvestnaya, O.M. 1987, Communications of the Special Astrophysical Observatory No. 55, Acad. Sci. URSS.

Kendall, M.G. 1957, "A Course of Multivariate Analysis" Griffin \& Co., London

502

T. J.-L. Courvoisier and A. Blecha: Multi-Wavelength Continuum Emission of AGN, 502.

(C) 1994 IAU. Printed in the Netherlands. 